\title{
A novel regulatory circuit of miR-152 and DNMT1 in human bladder cancer
}

\author{
HAIQING ZHANG ${ }^{1 *}$, DEFENG QI ${ }^{2 *}$, JINHUI LI $^{2,3^{*}}$, TAO PENG $^{2}$, LINQING YANG ${ }^{4}$, JIANHUI YUAN $^{4}$, \\ YUYING ZHANG ${ }^{1}$, YUAN HU ${ }^{2}$, JIALIN SU ${ }^{1,2}$, BIAO QUE ${ }^{1,2}$, MENGXI LI $^{2}$, GUOREN ZHOU $^{5}$, \\ ${\text { YUXIN } \mathrm{CHEN}^{6} \text {, WENJUAN ZHANG }}^{7}$ and WEIDONG JI ${ }^{1}$
}

\author{
${ }^{1}$ Center for Translational Medicine, The First Affiliated Hospital, Sun Yat-sen University, Guangzhou, Guangdong 510080; \\ ${ }^{2}$ Department of Urology, Minimally Invasive Surgery Center, Guangdong Key Laboratory of Urology, \\ The First Affiliated Hospital of Guangzhou Medical University, Guangzhou, Guangdong 510230; \\ ${ }^{3}$ The Affiliated Hospital of Medical School of Ningbo University, Nigbo, Zhejiang 315000; \\ ${ }^{4}$ Institute of Toxicology, Shenzhen Center for Disease Control and Prevention, Shenzhen, Guangdong 518055; \\ ${ }^{5}$ Department of Medical Oncology, Jiangsu Cancer Hospital, Nanjing, Jiangsu 210009; \\ ${ }^{6}$ Department of Laboratory Medicine, Nanjing Drum Tower Hospital, Nanjing, Jiangsu 210008; \\ ${ }^{7}$ Department of Toxicology, School of Medicine, Jinan University, Guangzhou, Guangdong 510632, P.R. China
}

Received February 14, 2018; Accepted July 4, 2018

DOI: $10.3892 /$ or. 2018.6553

\begin{abstract}
Downregulation of microRNA-152 (miR-152) has been observed in various types of human malignancies, including Bladder cancer (BC). However, the role of miR-152 in the development and progression of $\mathrm{BC}$ is still unclear. In our previous study, we identified a functional crosstalk between miR-152 and DNA methyltransferase 1 (DNMT1) involved in Nis-induced malignant transformation. In the present study, we found that the expression of miR-152 was specifically
\end{abstract}

Correspondence to: Professor Weidong Ji, Center for Translational Medicine, The First Affiliated Hospital, Sun Yat-sen University, Guangzhou, Guangdong 510080, P.R. China

E-mail:wdji2008@126.com

Dr Wenjuan Zhang, Department of Toxicology, School of Medicine, Jinan University, Guangzhou, Guangdong 510632, P.R. China E-mail: zhangwj11@126.com

${ }^{*}$ Contributed equally

Abbreviations: miR-152, microRNA-152; ATBC, adjacent tissues of bladder cancer; ATCC, American Type Culture Collection; BC, bladder cancer; ChIP, chromatin immunoprecipitation; DAC, 5-aza-2-deoxycytidine; DNMT1, DNA methyltransferase 1; DNMTs, DNA methyltransferases; IRB, The Institutional Review Board; m5C, 5-methylcytosine; MIBC, muscle-invasive bladder cancer; miRNAs, microRNAs; MLL, mixed-lineage leukemia; MSP, methylation-specific PCR; NMIBC, non-muscle-invasive bladder cancer; NSCLC, non-small cell lung cancer; TCGA, The Cancer Genome Atlas; 3'-UTR, 3' untranslated region

Key words: microRNA-152, DNA methylation, DNMT1, bladder cancer cells, regulatory circuit downregulated in BC cells and tissues via the DNA hypermethylation of the miR-152 promoter. The overexpression of miR-152 in BC cells resulted in a reduction of DNMT1, whereas the inhibition of the expression of miR-152 induced an elevated level of DNMT1. Further studies revealed that miR-152 directly downregulated the expression of DNMT1 by targeting the 3'-UTR of its transcript in BC cells. In addition, ectopic expression of miR-152 in BC cells significantly inhibited cell proliferation, whereas the inhibition of miR-152 expression led to increased cell proliferation. These findings indicated a novel regulatory circuit of miR-152/DNMT1 in $\mathrm{BC}$, and more importantly, the combination of miR-152 and DNMT1 may function as promising therapeutic modalities and early biomarkers for BC.

\section{Introduction}

Bladder cancer (BC) is a common malignancy worldwide. Approximately $70 \%$ of $\mathrm{BC}$ patients receive a preliminary diagnosis of non-muscle invasive $\mathrm{BC}$ (NMIBC). Of these, $50-70 \%$ of patients exhibit recurrence, and $10-20 \%$ progress to muscle-invasive BC (MIBC) (1). Over the last decade, significant progress has been made regarding the mechanisms, diagnosis and therapy of $\mathrm{BC}(2)$. Nonetheless, a high rate of recurrence of non-invasive $\mathrm{BC}$ and poor survival of patients with invasive $\mathrm{BC}$ have not been thoroughly solved. Therefore, there is an unmet need in understanding the molecular mechanisms of BC development, improving the effective treatment and early detection of disease recurrence.

MicroRNAs (miRNAs) are a group of non-coding small RNAs (22 nucleotides in length) that negatively regulate gene expression by binding to the 3 ' untranslated region (3'-UTR) of target mRNAs $(3,4)$. There is strong evidence supporting that the dysregulation of the expression of miRNAs is implicated in human cancer development and progression $(5,6)$. As one of the 
three members of the miR-148/152 family, miR-152 has been identified to accelerate growth of certain tumor types when its expression is downregulated (7). Recent studies reported that the aberrant expression of miR-152 plays an important role in the pathogenesis of $\mathrm{BC}(8,9)$. However, the molecular mechanism underlying the effect of aberrant expression of miR-152 on the development of $\mathrm{BC}$ remains poorly understood.

As other protein-coding genes, miRNA expression is regulated by the same mechanisms including epigenetic regulation $(10,11)$. As one of the most important epigenetic regulators, DNA methylation is involved in various biological processes including cancer (12-15). DNA methylation plays an important role in regulating gene expression, especially when it occurs in $\mathrm{CpG}$ island regions of gene promoters (16). Previous studies have indicated that several miRNAs are regulated by DNA methylation in various types of cancers and metabolic diseases (17-19). Notably, miRNAs control the chromatin structure by regulating histone modifiers and DNA methyltransferases such as DNA methyltransferases (DNMTs), ten-eleven translocations (TETs), histone deacetylases (HDACs) and polycomb genes, indicating that miRNAs can also indirectly modulate gene expression by controlling epigenetic modifications (20-22).

In our previous study, we identified a functional crosstalk between miR-152 and DNMT1 involved in Nis-induced malignant transformation (23). In the present study, we found that the expression of miR-152 in BC cells and tissues was relatively lower than in normal bladder cells and adjacent tissues of BC (ATBC), respectively. In addition, we identified a higher expression of DNMT1 in BC cells and tissues compared to normal bladder cells and adjacent tissues of BC (24). Additionally, methylation-specific PCR (MSP) revealed that miR-152 was methylated in BC cells and tissues, whereas unmethlylated miR-152 was present in normal bladder cells and adjacent tissues of BC. Since DNA methylation can be regulated by miRNAs by targeting DNMTs (25), we aimed to determine the presence of a regulatory circuit of miR-152 and DNMT1 in human BC. Furthermore, we also determined whether miR-152 contributed to tumor cell growth.

\section{Materials and methods}

Cell culture, treatment and transfection. The immortalized human bladder epithelial cell line SV-HUC-1 and the human urinary bladder transitional carcinoma cell line T24 were both purchased from the American Type Culture Collection (ATCC; Manassas, VA, USA). The human BC cell line UM-UC-3 was purchased from the Cell Bank of the Chinese Academy of Sciences (Shanghai, China). SV-HUC-1 cells were cultured in F12K, T24 cells were cultured in RPMI-1640 medium, and UM-UC-3 cells were cultured in MEM. All media were supplemented with $10 \%$ fetal bovine serum (FBS; Gibco; Thermo Fisher Scientific, Inc., Waltham, MA, USA), in a humidified air atmosphere of $5 \% \mathrm{CO}_{2}$ at $37^{\circ} \mathrm{C}$. Trypsin (0.25\% with $1 \mathrm{mM}$ EDTA) (Invitrogen, Carlsbad, CA, USA) was also used to harvest the cells for further experiments.

One day prior to transfection, the cells were plated in growth medium without antibiotics at a density of $50-60 \%$. miR-152 mimic and mimic-control were transfected into UM-UC-3 cells using Lipofectamine 2000 (Invitrogen) according to the manufacturer's protocol. miR-152 inhibitor and inhibitor-control were transfected into SV-HUC-1 cells as previously described (23). Cells were harvested at 24, 48, 72 and $96 \mathrm{~h}$ post-transfection. For combined treatment of the miR-152 inhibitor and the DNMT inhibitor, 5-aza-2-deoxycytidine (DAC), SV-HUC-1 cells transfected with miR-152 inhibitor were further divided into two groups, in which one group was treated with $12.5 \mu \mathrm{M}$ DAC (Sigma-Aldrich; Merck $\mathrm{KGaA}$ ) for $72 \mathrm{~h}$, and the other group was used as a negative control.

Patients and tissue samples. A set of 24 bladder tumor specimens was obtained from patients who underwent BC surgery between January 2012 and May 2015 at the First Affiliated Hospital of Guangzhou Medical University (Guangzhou, Guangdong, China). None of the patients received antitumor treatment prior to tumor sampling. A total of 24 adjacent tissues of bladder tumors from matched patients was collected as the control group. Details of the characteristics of the patients were described in our previous study (24). In order to further validate the relationship between miR-152 and DNMT1 mRNA expression, public data from both primary invasive and papillary BC tissues from 'The Cancer Genome Atlas' (TCGA) data portal (https://tcga-data.nci.nih.gov) were used, and explored through the cBio Cancer Genomics Portal (http://cbioportal.org). The data from 404 patients from Illumina HiSeq gene expression platforms (Illumina, Inc., San Diego, CA, USA) were extracted.

Real-time quantitative PCR. Total RNA from tissues or cell lines was extracted using TRIzol reagent (Invitrogen) according to the manufacturer's instructions. The quantity and quality of the RNA were determined by UV spectrophotometer. Bulge-loop ${ }^{\mathrm{TM}}$ miRNA qRT-PCR Primer sets (one RT primer and a pair of qPCR primers for each set) specific for miR-152 were designed by Guangzhou RiboBio Co., Ltd. (Guangzhou, China). The relative quantification value of the target, normalized to the control, was calculated by the the $\Delta \Delta$ Cq method (26). PCR was carried out as follows: $95^{\circ} \mathrm{C}$ for $20 \mathrm{sec}, 40$ cycles of $10 \mathrm{sec}$ at $95^{\circ} \mathrm{C}, 20 \mathrm{sec}$ at $58^{\circ} \mathrm{C}$ and $30 \mathrm{sec}$ at $72^{\circ} \mathrm{C}$. Samples were analyzed in triplicate.

Western blot analysis. Protein was extracted from T24, UM-UC-3 and SV-HUC-1 cells for western blotting. Cultured cells were collected and washed three times with PBS. Following incubation with RIPA buffer (Sigma-Aldrich; Merck KGaA) containing $5 \mathrm{mM}$ EDTA, PMSF, cocktail inhibitor and phosphatase inhibitor cocktail, cells were collected in a centrifuge tube. Cell lysates were centrifuged at $13,000 \mathrm{x} \mathrm{g}$ for $15 \mathrm{~min}$ at $4^{\circ} \mathrm{C}$ and insoluble debris was discarded. The proteins were determined by BCA quantitative method. Soluble proteins $(30 \mu \mathrm{g})$ were subjected to $8 \%$ SDS-PAGE and transferred to a Hybond-P polyvinylidene difluoride $(P V D F)$ membrane. Membranes were blocked in TPBS (PBS with $0.05 \%$ Tween-20) containing 5\% (w/v) non-fat dry milk for $1 \mathrm{~h}$ at room temperature, washed in TPBS and then incubated with primary antibody. Immunoblottings were performed with 1:1,000 diluted anti-DNMT1 antibody (cat. no. D4692; Sigma-Aldrich; Merck KGaA) and 1:1,000 diluted anti-GAPDH antibody (cat. no. sc-166545; Santa Cruz 
Table I. Primers used in this study.

Primer sequence (5'-3')

Primer name

F

$\mathrm{R}$

\begin{abstract}
MSP
miR-152 (methylated)

miR-152 (non methylated)

\section{TTTAGAATTCGCGCGTTC \\ GGAGGAAAAGTTTGTTTTAGTGTT}

ChIP

miR-152 promoter
AGAGGAGGCCTGTCCTGAGT
CCGACGAACTCAAAACAAA

ACCCACCAATAATAAAAACCAAA

F, forward; R, reverse.

Biotechnology, Santa Cruz, CA, USA). Blots were visualized using the Immobilon solutions (EMD Millipore, Billerica, MA, USA) under a chemiluminescence detection system, the ChemiDoc XRS (Bio-Rad Laboratories). Band area intensity was analyzed using Quantity One software (Bio-Rad Laboratories).

Methylation-specific PCR (MSP). Genomic DNA of tissues and cells was extracted using standard phenol/chloroform purification and ethanol precipitation. Bisulfite modification of the genomic DNA was performed using EZ DNA Methylation-Gold kit (Zymo Research Corp., Irvine, CA, USA). Methylation of each sample was evaluated in triplicate using MSP. PCR products were electrophoresed on a $2 \%$ agarose gel for analysis. Primer sequences for miR-152 are listed in Table I.

Chromatin immunoprecipitation (ChIP). Chromatin immunoprecipitation was performed using EZ-Magna ChIP A/G kit (EMD Millipore) according to the manufacturer's instructions. Briefly, cells were grown on $100-\mathrm{mm}$ plates to $85 \%$ confluence; formaldehyde was added to a final concentration of $1 \%$, and the plates were incubated for $10 \mathrm{~min}$ at $37^{\circ} \mathrm{C}$; the cross-linking reaction was stopped by the addition of $0.125 \mathrm{M}$ glycine for $5 \mathrm{~min}$ at room temperature. Cells were sonicated and sheared to yield fragments between 200 and 1,000 bp. Anti-DNMT1 (5 $\mu \mathrm{g}$; Abcam, Cambridge, UK) was added to the sonicated samples and incubated at $4^{\circ} \mathrm{C}$ overnight with rotation. Immune complexes were collected with Protein $\mathrm{A} / \mathrm{G}$ agarose beads and washed with buffer to remove nonspecific binding. Protein/DNA complex was reverse crosslinked and DNA was purified using spin columns. DNA was detected with quantitative PCR. Primers for ChIP-qPCR are listed in Table I.

Cell proliferation analysis. The miR-152 mimic was transfected into the T24 and UM-UC-3 cells and the miR-152 inhibitor was transfected into the SV-HUC-1 cells lasting $6 \mathrm{~h}$. Subsequently, the cells were plated into a new dish. Cells $\left(2 \times 10^{3}\right)$ were plated in triplicate and measured at the indicated time-points: 24, 48, 72 and $96 \mathrm{~h}$. The number of cells was determined using the CellTiter $96^{\circledR}$ AQueous One Solution assay (Promega Corp., Madison, WI, USA). Triplicate plates were counted for each cell line.
Luciferase reporter assay. The cells $\left(1 \times 10^{5}\right.$ cells/well) were seeded in 24-well plates. When cells reached $70-80 \%$ confluence, $0.5 \mu \mathrm{g}$ of reporter plasmids alone or co-transfected with or without microRNA mimics were transiently transfected using Lipofectamine 2000 according to the manufacturer's instructions (Invitrogen; Thermo Fisher Scientific, Inc.). Luciferase activity was determined as previously reported (23).

Statistical analysis. All analyses were performed with the SPSS 13 statistical software (SPSS, Inc., Chicago, IL, USA). Statistical significance was determined by Student's t-test and Mann-Whitney U test. The relationship between the expression level of miR-152 and clinicopathological parameters was analyzed using the Mann-Whitney U test. The correlations were analyzed using Spearman's correlation rank test. Differences in probability values (P-values) of $<0.05$ were considered to indicate a statistically significant difference.

\section{Results}

miR-152 is downregulated in BC cells and tumor tissues. To determine the expression of miR-152 in BC, real-time PCR was used to compare the expression levels of miR-152 in T24 cells, UM-UC-3 cells and SV-HUC-1 cells. A lower level of miR-152 was observed in T24 cells and UM-UC-3 cells compared with SV-HUC-1 cells (Fig. 1A). We further compared the expression of miR-152 between 24 BC tissue specimens and the matched adjacent normal tissues. Consistent with our observations in cells, compared to the matched adjacent tissues of BC, miR-152 had a relatively lower expression in BC tissues (Fig. 1B). Additionally, after transfection with the miR-152 mimic, the expression of miR-152 in T24 cells had an increased level in a time-dependent manner (Fig. 1C). Conversely, treatment with miR-152 inhibitor in SV-HUC-1 cells resulted in a reduction of miR-152 expression in a time-dependent manner (Fig. 1D). These results indicated that miR-152 was downregulated in BC cell and tumor tissues. Notably, the analysis of the clinicopathological features of $\mathrm{BC}$ patients revealed that a lower miR-152 expression level was significantly correlated with the stage and grade of $\mathrm{BC}$ patients, which was independent of patient sex and age (Table II). 

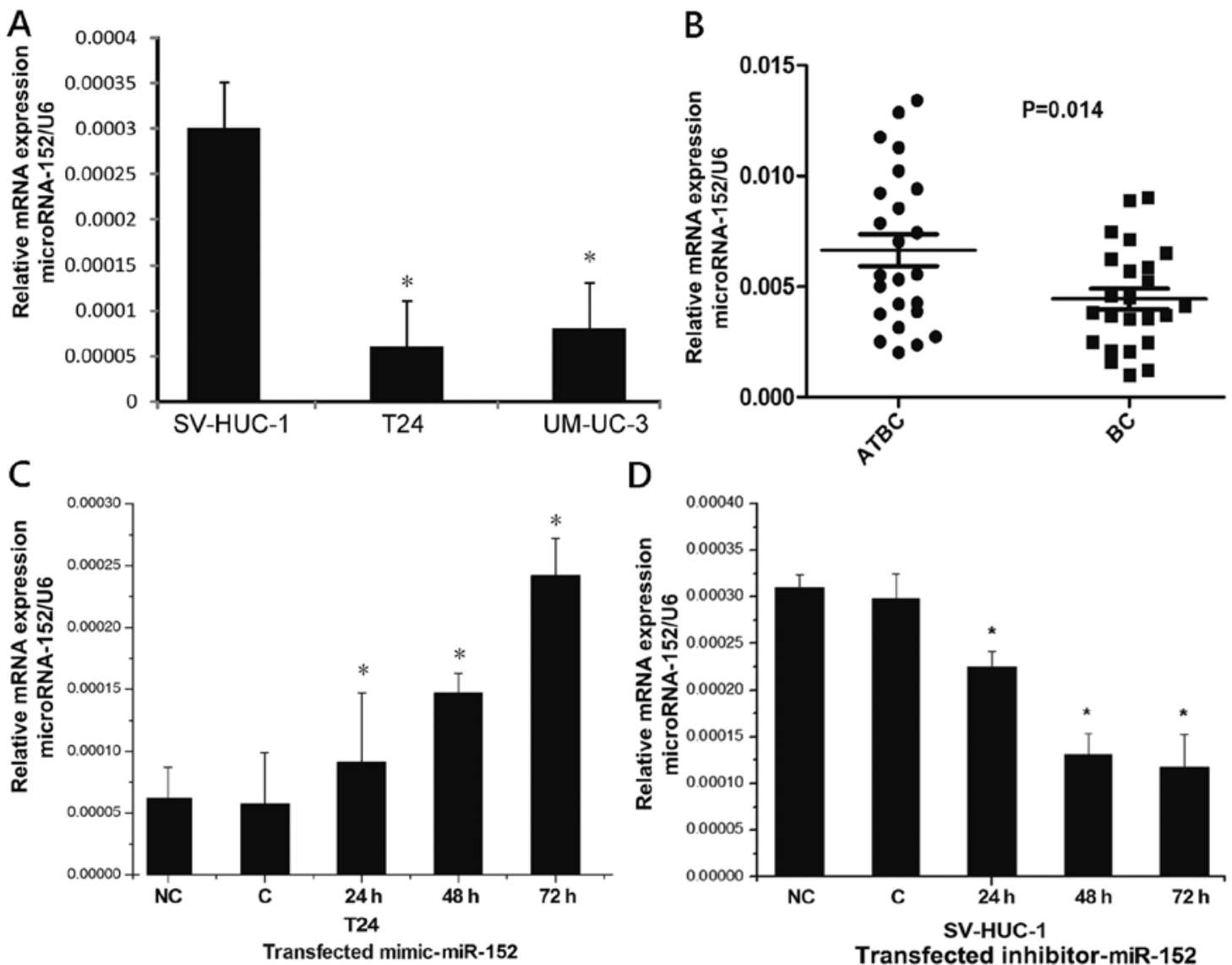

Figure 1. Downregulation of miR-152 in BC cells and bladder tumor tissues. (A) Levels of miR-152 expression in T24, SV-HUC-1 and UM-UC-3 cells were determined by real-time PCR with input normalization by U6 snRNA level. Results represent the mean \pm SD from three independent experiments. ${ }^{*} \mathrm{P}<0.05$, compared to SV-HUC-1 cells. (B) Relative expression levels of miR-152 in 24 pairs of BC tumors and the ATBT were determined by real-time PCR with input normalization by U6 snRNA level. (C) The expression of miR-152 in T24 cells transfected with miR-152 mimics or negative control. "P<0.05, compared with the negative control group. (D) The expression of miR-152 in SV-HUC-1 cells transfected with miR-152 inhibitor or negative control. " $\mathrm{P}<0.05$, compared with the negative control group. miR-152, microRNA-152; BC, bladder cancer; snRNA, small nuclear RNA; ATBT, matched adjacent tissues of BC.

Table II. Clinicopathological features of BC patients and the levels of miR-152 expression in cancer tissues.

Molecular expression level

Characteristics

No. of patients $(\%)$

$\operatorname{miR}-152^{\mathrm{c}}$

P-value

\section{All patients}

Sex ${ }^{\mathrm{a}}$

Females

Males

Age at diagnosis ${ }^{\mathrm{a}}$, years

$\begin{array}{ll}\leq 70 & 12(50.0) \\ >70 & 12(50.0)\end{array}$

Pathological grade ${ }^{\mathrm{a}}$

Low

High

Pathological stage ${ }^{b}$
pTa
pT1
$\geq \mathrm{pT} 2$

0.725

$0.0046(0.0016-0.0088)$

$0.0042(0.001-0.0089)$

0.0037 (0.001-0.0074)

$0.0051(0.0012-0.0089)$

$0.0064(0.0035-0.0089)$

$0.0034(0.0010-0.0062)$

$0.0061(0.0045-0.0088)$

$0.0045(0.0012-0.0089)$

0.0027 (0.001-0.0038)

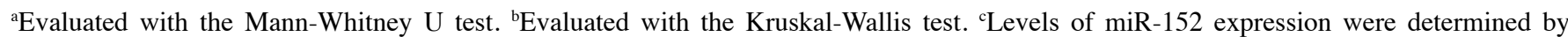
qRT-PCR assay, and normalized to the U6 levels using the $\Delta \Delta \mathrm{Cq}$ method (26). ${ }^{\mathrm{d}}$ Significant difference. BC, bladder cancer. 
A

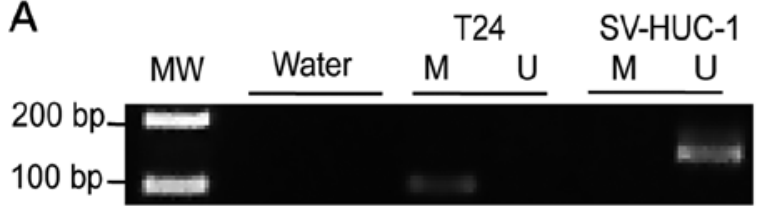

C

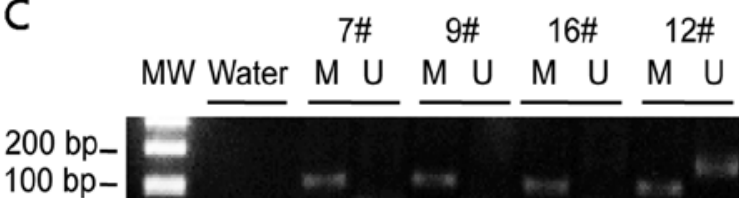

E
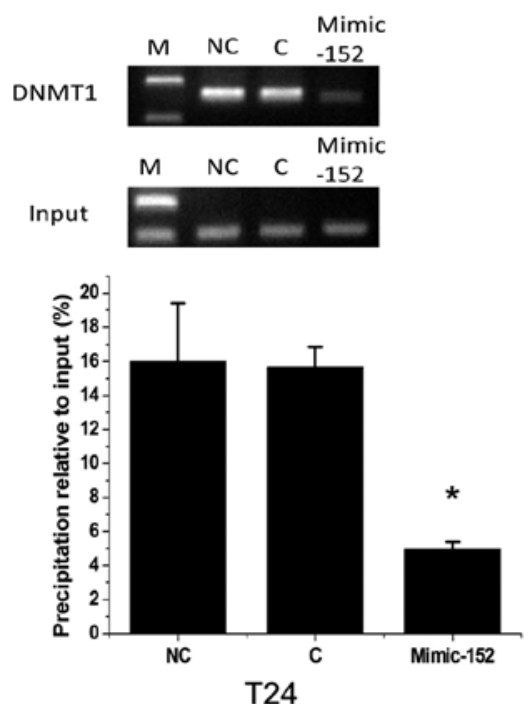

B

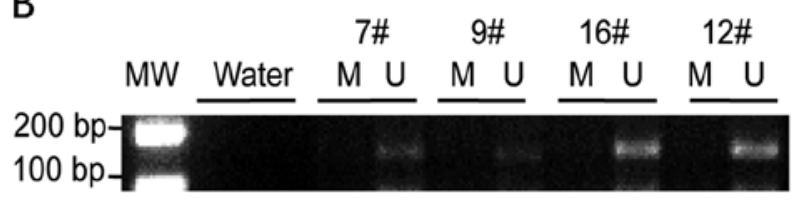

D

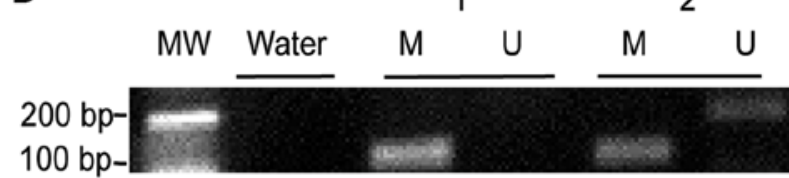

$\mathrm{F}$

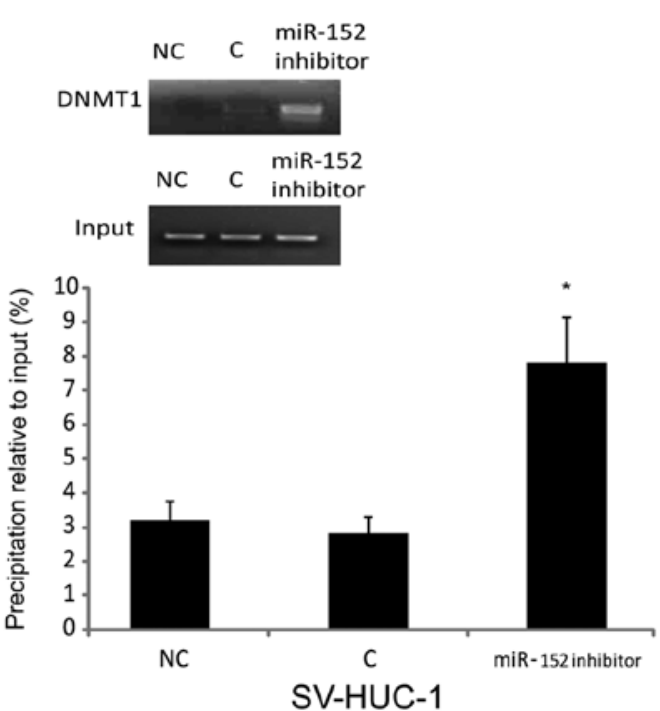

Figure 2. The expression of miR-152 was regulated by DNA methylation via DNMT1. (A) MSP analysis of miR-152 gene promoter in T24 and SV-HUC-1 cells. MSP, methylation-specific PCR; MW, molecular weight; U, unmethylated status; M, methylated status. Water was used as a control for PCR. (B) MSP analysis of miR-152 gene promoter in BC tumors. MW, molecular weight; $7^{\sharp}, 9^{\#}, 16^{\#}, 12^{\#}$, Number of tumor samples. (C) MSP analysis of miR-152 gene promoter in the matched adjacent tissues of BC. MW, molecular weight; $7^{\#}, 9^{\#}, 16^{\#}, 12^{\#}$, Number of adjacent tissues samples. (D) MSP analysis of miR-152 gene promoter in T24 cells transfected with miR-152 mimics and control. MW, molecular weight; 1, Control; 2, T24 mimic-152. (E) The ChIP analysis of the binding of DNMT1 in miR-152 CpG island promoter in T24 cells transfected with miR-152 mimics and control. Quantitative-ChIP analysis was conducted to assess the binding of DNMT1 in miR-152 CpG island promoter with specific antibodies of DNMT1 with normalization by total input DNA. Results represent the mean \pm SD from three independent experiments. " $\mathrm{P}<0.05$, compared with the negative control group. Right images are representative results from quantitative PCR after ChIP assay. M, marker; NC, negative control; C, control cells; ChIP, Chromatin immunoprecipitation. (F) The ChIP analysis of the binding of DNMT1 in miR-152 CpG island promoter in SV-HUC-1 cells transfected with miR-152 inhibitor and control. ${ }^{*} \mathrm{P}<0.05$, compared with the negative control group. miR-152, microRNA-152; DNMT1, DNA methyltransferase 1; BC, bladder cancer.

Downregulation of miR-152 is due to DNA hypermethylation via DNMT1 in BC. The reduction or absence of gene expression mediated by DNA hypermethylation plays an important role in cancer development (27). We hypothesized that downregulation of miR-152 in BC was due to DNA methylation. To verify our hypothesis, we used an MSP method to determine the methylation status of miR-152 promoter in T24 and SV-HUC-1 cells. We observed that miR-152 was methylated in T24 cells, but not in SV-HUC-1 cells (Fig. 2A). Similarly, 17 out of $24(70.8 \%)$ samples had CpG island methylation of miR-152 promoter in BC tissues, while only 9 out of $24(37.5 \%)$ samples had $\mathrm{CpG}$ island methylation of miR-152 promoter in the matched normal tissues adjacent to BC (Fig. 2B and C). Conversely, our previous study demonstrated that the expression level of DNMT1 was significantly higher in BC cells and tissues (24). To explore the potential regulatory circuit of DNMT1 and miR-152, we transfected miR-152 mimics in T24 cells and detected the expression of DNMT1 and the methylation level of miR-152 CpG island promoter. We determined that both DNMT1 mRNA expression and the
DNA methylation of miR-152 promoter were decreased in T24 cells transfected with miR-152 mimics (Figs. 3B and 2D). Notably, the binding of DNMT1 to the miR-152 CpG island promoter was significantly decreased in T24 cells transfected with miR-152 mimics (Fig. 2E). In addition, DNMT1 binding to the miR-152 CpG island promoter was significantly increased in SV-HUC-1 cells transfected with the miR-152 inhibitor compared to the control group (Fig. 2F). To further determine the relationship between the expression of miR-152 and DNMT1, we performed Spearman's rank correlation analysis and found a significantly negative correlation between the expression levels of miR-152 and DNMT1 in BC specimens ( $\mathrm{r}=-0.623, \mathrm{P}=0.01$ ) (Fig. 3A). In order to further confirm these findings, we determined DNMT1 and miR-152 expression in The Cancer Genome Atlas (TCGA) dataset which includes a total of $404 \mathrm{BC}$ samples. Notably, we also identified a negative correlation $(r=-0.145)$ in $B C$ samples of stage IV, although there was no correlation of DNMT1 with miR-152 mRNA expression among BC samples of all stages. In addition, miR-152 levels may affect disease-free 
A

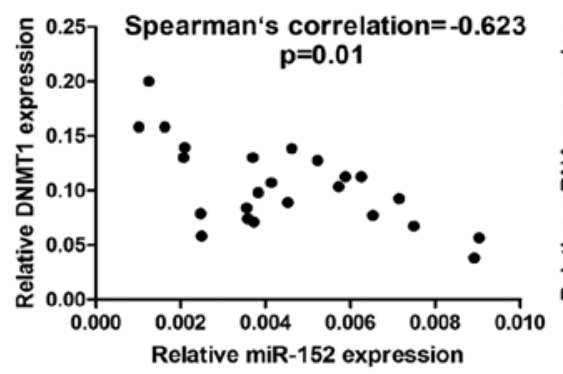

D

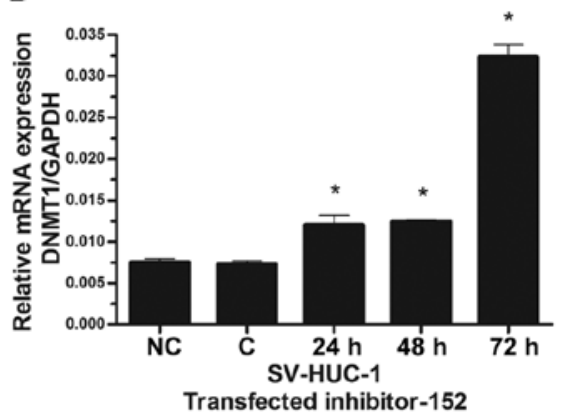

G

SV-HUC-1 inhibitor-152

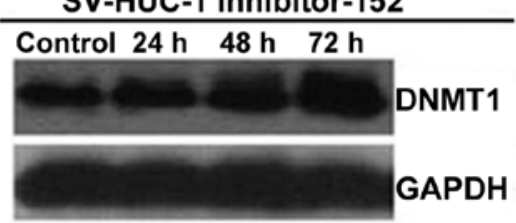

B

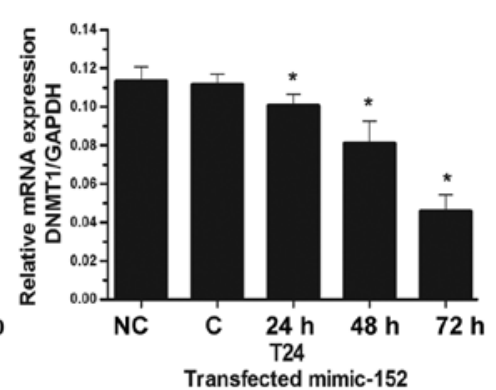

$\mathrm{E}$

T24 mimic-152

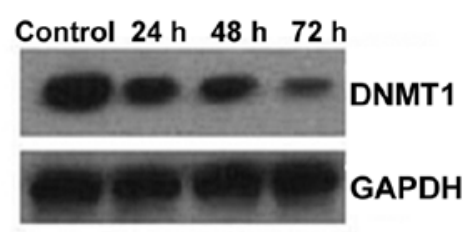

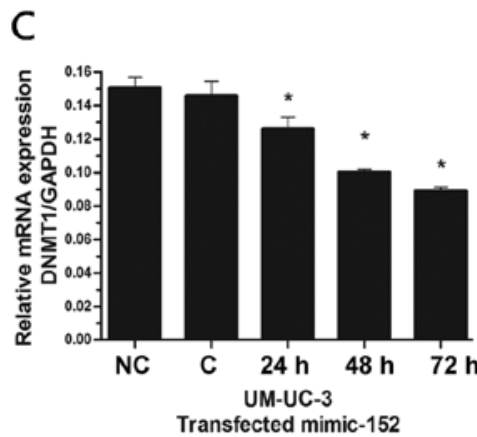

F

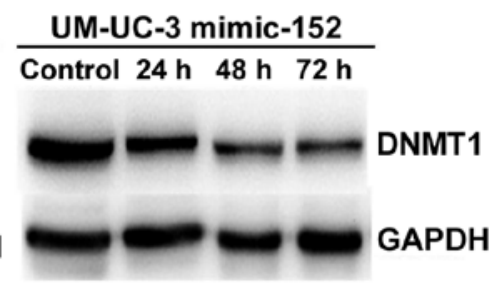

Figure 3. The expression of DNMT1 is regulated by miR-152. (A) Association of DNMT1 mRNA and miR-152 mRNA expression in 24 primary BC samples. $r$, Spearman correlation coefficient. (B) The expression of DNMT1 in T24 transfected with miR-152 mimics or negative control, respectively. ${ }^{*}<0.05$, compared with the negative control group. (C) The expression of DNMT1 in UM-UC-3 cells transfected with miR-152 mimics or negative control. * $<<0.05$, compared with the negative control group. (D) The expression of DNMT1 in SV-HUC-1 cells transfected with the miR-152 inhibitor or negative control. ${ }^{*} \mathrm{P}<0.05$, compared with the negative control group. (E) DNMT1 protein expression in T24 cells transfected with miR-152 mimics. (F) DNMT1 protein expression in UM-UC-3 cells transfected with miR-152 mimics. (G) DNMT1 protein expression in SV-HUC-1 cells transfected with the miR-152 inhibitor or negative control. miR-152, microRNA-152; DNMT1, DNA methyltransferase 1; BC, bladder cancer.

survival $(\mathrm{P}=0.026)$ (data not shown). Collectively, our results indicated that hypermethylation via DNMT1 is responsible for the silencing of miR-152 expression in BC.

Expression of DNMT1 is regulated by $\mathrm{miR}-152$ by targeting the 3 '-UTR in BC cells. DNMT1 is the key enzyme in DNA methylation which has been identified as one of the high-scoring candidate genes of miR-152 targets (28). To further determine whether the expression of DNMT1 is regulated by miR-152, we first determined the levels of DNMT1 mRNA in T24 and UM-UC-3 cells transfected miR-152 mimics by real-time PCR. We found that DNMT1 expression was decreased in both of T24 and UM-UC-3 cells transfected with the miR-152 mimic in a time-dependent manner (Fig. 3B and C). Conversely, the level of DNMT1 mRNA in SV-HUC-1 cells transfected the miR-152 inhibitor was increased in a time-dependent manner (Fig. 3D). These results were ascertained by western blot analysis (Fig. 3E-G). Notably, we revealed that miR-152 could directly target DNMT1 3'-UTR and suppress its expression in NiS-transformed cells (23). To further validate our results in T24 cells and SV-HUC-1 cells, a luciferase reporter assay was performed, in which miR-152 significantly reduced DNMT1 wild-type 3'-UTR luciferase activity, but not DNMT1

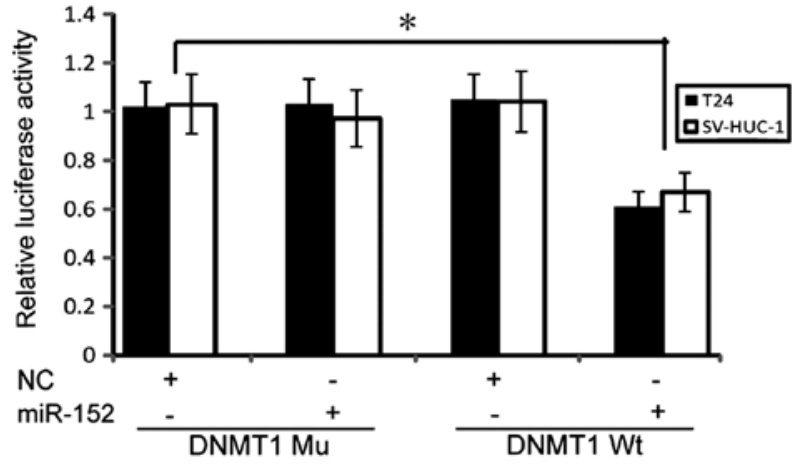

Figure 4. miR-152 suppresses DNMT1 expression by targeting the 3' UTR in BC cells. A dual-luciferase assay was performed in T24 cells and SV-HUC-1 cells co-transfected with the firefly luciferase constructs containing the DNMT1 WT or Mu 3'-UTR and miR-152 mimics or scrambled oligonucleotides as the negative control. ${ }^{*} \mathrm{P}<0.05$, compared with cells transfected with the negative control. Wt, wild-type; Mu, mutant; miR-152, microRNA-152; DNMT1, DNA methyltransferase 1; BC, bladder cancer.

Mu 3'-UTR activity (Fig. 4), indicating that miR-152 directly bound to the DNMT1 3'-UTR and inhibited its expression in $\mathrm{BC}$ cells. 

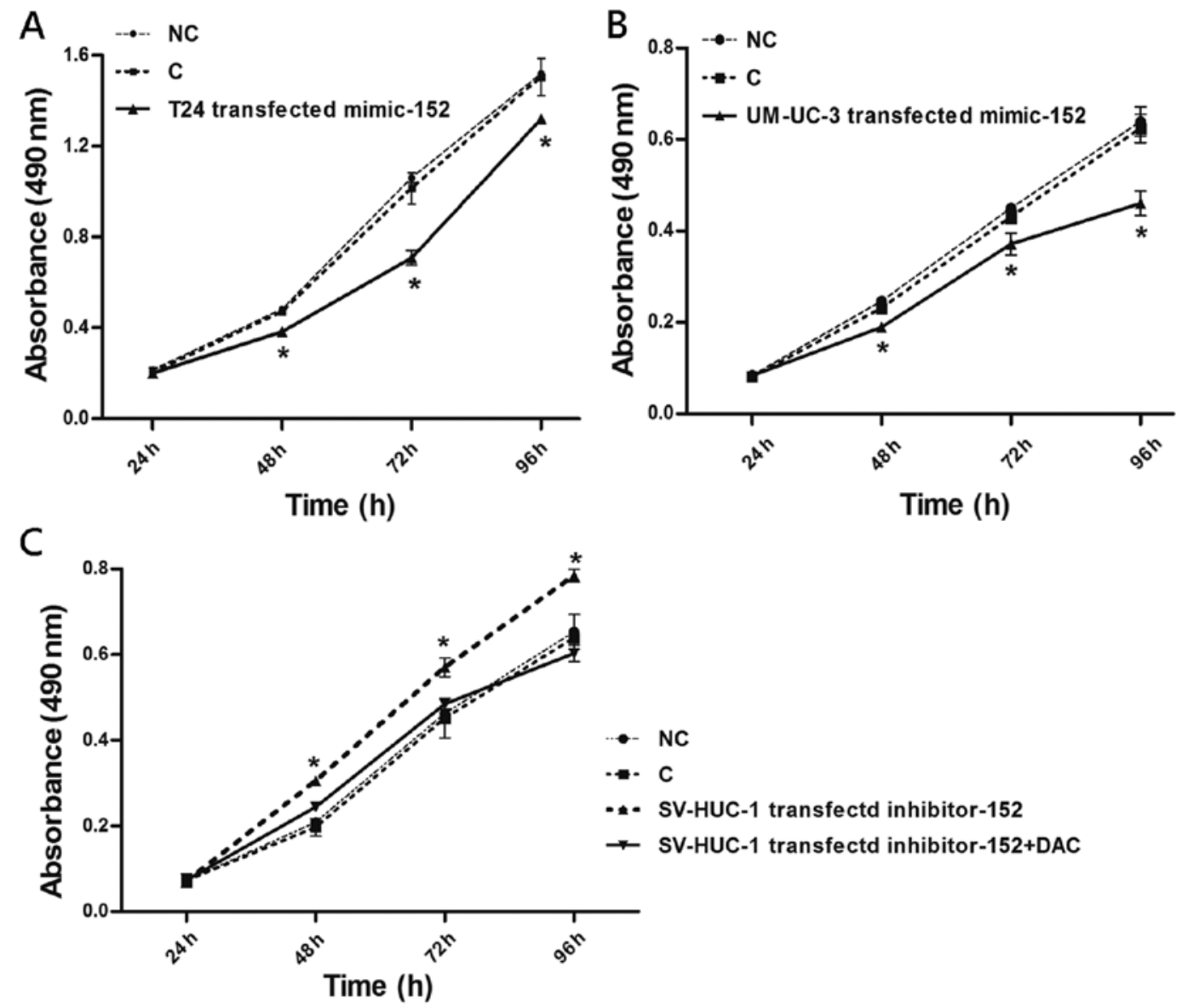

Figure 5. miR-152 inhibits cell growth. (A) The absorbance at $490 \mathrm{~nm}$ was assessed each day for $96 \mathrm{~h}$ in BC T24 cells transfected with or without miR-152 mimics or the negative control. (B) The absorbance at $490 \mathrm{~nm}$ was determined each day for $96 \mathrm{~h}$ in UM-UC-3 cells transfected with or without miR-152 mimics or the negative control. (C) The absorbance at $490 \mathrm{~nm}$ was assessed each day for $96 \mathrm{~h}$ in SV-HUC-1 cells transfected with or without miR-152 inhibitor or the negative control, and combined treatment of miR-152 inhibitor and DNMT inhibitor (DAC). The data are expressed as the mean \pm SEM $(n=3)$. ${ }^{*} \mathrm{P}<0.05$, compared with cells transfected with the negative control. miR-152, microRNA-152; BC, bladder cancer.

miR-152 inhibits BC cell growth. In order to clarify whether the expression of miR-152 plays a significant role in BC malignant phenotypes, we explored the effects of ectopically expressed miR-152 on the proliferation of T24, UM-UC-3 and SV-HUC-1 cells. Both T24 and UM-UC-3 cells transfected with miR-152 mimics exhibited significantly decreased cell proliferation at 48, 72 and $96 \mathrm{~h}$, compared to the miR-control (Fig. 5A and B). Conversely, SV-HUC-1 cells transfected with miR-152 inhibitor had increased cell proliferation at 48, 72, and $96 \mathrm{~h}$, compared to the miR-control (Fig. 5C). Notably, the combined treatment of the miR-152 inhibitor and the DNMT inhibitor, 5-aza-2-deoxycytidine (DAC), led to decreased cell proliferation compared to suppression of the miR-152 inhibitor alone, whereas the combined treatment resulted in a slight elevation of cell proliferation compared to that in control SV-HUC-1 cells at 48 and $72 \mathrm{~h}$ post treatment. These findings highlighted a reciprocal regulatory loop between miR-152 and DNMT1.

\section{Discussion}

In recent years, there has been an increasing interest in the role of epigenetic regulation in human diseases (29). For instance, numerous studies have revealed that epigenetic dysregulation of miRNA expression plays a key role in cancer development $(30,31)$. In the present study, we demonstrated that upregulation of DNMT1 was responsible for the hypermethylation of miR-152, which resulted in the downregulation of miR-152. miR-152 mediated DNA methylation by directly targeting DNMT1 3'-UTR, indicative of a novel regulatory circuit between DNMT1 and miR-152 in human BC.

MiRNAs are a group of post-transcriptional regulators which inhibit target gene expression by mRNA degradation and translation inhibition $(32,33)$. Several studies have revealed that miRNAs are altered in many cancers, and that they can initiate carcinogenesis or drive tumor progression $(11,34)$. miR-152 is located at $17 \mathrm{q} 21.32$, and a typical $\mathrm{CpG}$ island is surrounded by miR-152 promoter. Huang et al have suggested a tumor-suppressive role of miR-152 in the epigenetic aberration of HBV-related hepatocellular carcinoma (25). Stumpel et al (35) reported that downregulation of miR-152 was related to $\mathrm{CpG}$ hypermethylation. Further research indicated that the hypermethylation of the miR-152 CpG island was associated with poor clinical outcome in mixed-lineage leukemia (MLL). In BC, Kohler et al (9) reported that a reduced expression of miR-152 was related to increased DNA methylation. Consistent with this study, our results demonstrated the downregulation of miR-152 expression in $\mathrm{BC}$ as well as the hypermethylation at the promoter region of miR-152. Notably, 
we identified that the DNMT1 bound to the miR-152 CpG island promoter was markedly decreased in T24 cells transfected with miR-152 mimics, which was similar to a previous study revealing that the overexpression of miR-152 decreased DNMT1 and MeCP2 binding to the CpG island promoter of miR-152 in an FLS model (36). In addition, it was revealed that miR-152 could act as a tumor suppressor by targeting TGF, and inhibit prostate cancer cell migration and invasion (37). Overexpression of miR-152 suppressed cell proliferation and colony formation in non-small-cell lung cancer (NSCLC) cells by inhibiting FGF2 (38). Furthermore, miR-152 was downregulated in human glioblastoma stem cells (GSCs). Restoring the expression of miR-152 inhibited the cell proliferation of GSCs by downregulating KLF4 (39). Recently, miR-152 was identified as a regulator of lung metastasis which increased metastasis rates, but failed to promote primary tumor growth through a genome-wide in vivo CRISPR/Cas9 screening (40). Consistent with these studies, we also found that the proliferation of T24 cells and UM-UC-3 cells was significantly inhibited after treatment with miR-152 mimics. Conversely, the proliferation of SV-HUC-1 cells transfected with the miR-152 inhibitor was significantly increased, compared to those transfected with the inhibitor miR-control. Collectively, our results further confirmed the tumor-suppressive activity of miR-152 which is epigenetically silenced during human BC development.

The downregulation of miR-152 mediated by the methylation of miR-152 promoters was associated with breast cancer grades and lymph node-metastasis (LN) status (41). Similarly, lower expression of miR-152 was related to increased tumor sizes and advanced tumor stages in gastric cancer $(7,42)$. In addition, DNA hypermethylation on $\mathrm{CpG}$ islands was related to the overexpression of DNMT1 in multistage bladder carcinogenesis (43). In accordance with these findings, our results demonstrated that the upregulation of DNMT1 and downregulation of miR-152 were related to the grades and stages of BC.

Epigenetic dysfunction of genes is an important feature in human cancer development. Increasing evidence has indicated that DNA methylation is one of the key regulators in urinary tumors. DNMT1 plays a vital role in human cell malignant transformation, indicating that the aberrant expression of DNMT1 may contribute to the development of human cancer (44). Our present study revealed that the high expression of DNMT1 expression was correlated with low expression of miR-152 with hypermethylation of miR-152 promoter in $\mathrm{BC}$ cells and tissues. Furthermore, the upregulation of miR-152 expression led to decreased expression of DNMT1. All these findings indicated a dysregulated DNMT1 expression due to aberrant expression of miR-152 in BC.

Previous findings revealed that miR-152 induced aberrant DNA methylation in hepatitis B virus-related hepatocellular carcinoma (25) and ovarian cancer by targeting DNA methyltransferase 1 (45). In an arthritic rat model, miR-152 was found to regulate canonical Wnt pathway activation by targeting DNMT1 3'-UTR (46). Consistent with these studies, our results indicated that DNMT1 expression was suppressed by miR-152 via a direct interaction with a highly conserved region of the DNMT1 3'UTR in BC. Collectively, these findings demonstrated that miR-152 directly interacted with DNMT1 by targeting its predicted binding site in BC.
Notably, the relationship between miRNA-epigenetics is enriched by a line of findings indicating that the expression levels of the epigenetic effectors can be regulated by some miRNAs, while epigenetic effectors can also modulate the expression of these miRNAs $(47,48)$. For example, in pancreatic cancer, the overexpression of miR-148b and miR-152 repressed the expression of DNMT1, leading to the decreased DNA methylation and overexpression of tumor-suppressor genes (49). The overexpression of miR-152 in dairy cow mammary epithelial cells resulted in significantly lowered expression of DNMT1, which in turn led to a reduction of global DNA methylation (50). Conversely, miRNAs can also be modified by DNMTs. The putative $\mathrm{CpG}$ island promoter region of $\mathrm{miR}-29 \mathrm{~b} / \mathrm{c}$ was revealed to be regulated by DNA methylation. The increased expression of DNMT3a was related to silencing of miR-29b/c in gastric carcinogenesis (51). In addition, the overexpression of DNMT1 led to the hypermethylation of miR-148a and miR-152 genes in breast cancer. Furthermore, the expression of DNMT1, a direct target of miR-148a and miR-152, was inversely correlated with the expression levels of miR-148a/152, indicating a negative feedback regulatory loop between miR-148a/152 and DNMT1 (41). In a previous study, we identified a crucial functional crosstalk between miR-152 and DNMT1 via a double-negative circuit involved in NiS-induced malignant transformation (23). In the present study, we confirmed that a high miR-152 level was associated with low DNMT1 expression in SV-HUC-1 cells, whereas a low level of miR-152 was related to the high DNMT1 protein expression in T24 cells and UM-UC-3 cells, indicating a negative feedback regulation between miR-152 and DNMT1 expression in $\mathrm{BC}$.

In conclusion, we identified a novel regulatory circuit between miR-152 and DNMT1 and our study revealed that the tumor-suppressive activity of miR-152 is epigenetically silenced by directly interacting with the DNMT1 in BC. The discovery of the relationship between miR-152 and DNMT1 is highly beneficial to enrich our understanding of BC development, which could be further explored as effective therapeutic targets and early detection biomarkers.

\section{Acknowledgements}

Not applicable.

\section{Funding}

The present study was partly supported by grants from The National Natural Science Foundation of China (nos. 81472999, 81772699, 81272350 to WJ; no. 81473014 to WZ and no. 81600201 to YC), The Key Natural Science Foundation of Guangdong (no. 2015A030311038 to WJ) and Guangzhou People's Livelihood Science and Technology Project (no. 201803010052 to WJ), The Program of Health Department of Guangdong Province (no. C2013025 to DQ), The Key Science Foundation of Guangzhou (no. 201804020023 to DQ), The Six Talent Program of Jiangsu (no. 2013WSW-035 to GZ), The Research Plan of Discipline Distribution Program of Shenzhen (JCYJ20160328161613864 to JY) and The Technology Research Plan of Discipline Distribution Program of Shenzhen (JSGG20170414104216477 to JY). 


\section{Availability of data and materials}

The datasets used during the present study are available from the corresponding author upon reasonable request.

\section{Authors' contributions}

HZ, DQ and JL conceived and performed the experiments and analyzed the data. TP, LY, JY, YZ, GZ and YC performed the experiments. YH, JS, BQ and ML collected the clinical data and specimens. WJ and $\mathrm{WZ}$ conceived the project, analyzed the data, wrote the manuscript and provided supervision. All authors read and approved the manuscript and agree to be accountable for all aspects of the research in ensuring that the accuracy or integrity of any part of the work are appropriately investigated and resolved.

\section{Ethics approval and consent to participate}

The present study was performed in accordance with the Declaration of Helsinki. Written informed consent was obtained from each patient enrolled in this study. The present study was reviewed and approved by the Ethics Committee of Guangzhou Medical University in November 2015 (no. 2015-11).

\section{Patient consent for publication}

Not applicable.

\section{Competing interests}

The authors declare that they have no competing interests.

\section{References}

1. Kaufman DS, Shipley WU and Feldman AS: Bladder cancer. Lancet 374: 239-249, 2009.

2. Cancer Genome Atlas Research N: Comprehensive molecular characterization of urothelial bladder carcinoma. Nature 507: 315-322, 2014.

3. Ambros V: The functions of animal microRNAs. Nature 431: 350-355, 2004

4. Bartel DP: MicroRNAs: Genomics, biogenesis, mechanism, and function. Cell 116: 281-297, 2004.

5. Chen Q, Chen X, Zhang M, Fan Q, Luo, S and Cao X: miR-137 is frequently downregulated in gastric cancer and is a negative regulator of Cdc42. Dig Dis Sci 56: 2009-2016, 2011.

6. Croce CM: Causes and consequences of microRNA dysregulation in cancer. Nat Rev Genet 10: 704-714, 2009.

7. Chen Y, Song Y, Wang Z, Yue Z, Xu H, Xing C and Liu Z: Altered expression of MiR-148a and MiR-152 in gastrointestinal cancers and its clinical significance. J Gastrointest Surg 14: 1170-1179, 2010.

8. Jiang X, Du L, Wang L, Li J, Liu Y, Zheng G, Qu A, Zhang X, Pan $\mathrm{H}$, Yang Y, et al: Serum microRNA expression signatures identified from genome-wide microRNA profiling serve as novel noninvasive biomarkers for diagnosis and recurrence of bladder cancer. Int J Cancer 136: 854-62, 2015.

9. Kohler CU, Bryk O, Meier S, Lang K, Rozynek P, Brüning T and Käfferlein HU: Analyses in human urothelial cells identify methylation of miR-152, miR-200b and miR-10a genes as candidate bladder cancer biomarkers. Biochem Biophys Res Commun 438: 48-53, 2013.

10. Davalos V and Esteller M: MicroRNAs and cancer epigenetics: A macrorevolution. Curr Opin Oncol 22: 35-45, 2010.
11. Rouhi A, Mager DL, Humphries RK and Kuchenbauer F: MiRNAs, epigenetics, and cancer. Mamm Genome 19: 517-525, 2008.

12. Davis CD and Uthus EO: DNA methylation, cancer susceptibility, and nutrient interactions. Exp Biol Med 229: 988-995, 2004.

13. Jones PA: DNA methylation errors and cancer. Cancer Res 56: 2463-2467, 1996.

14. Laird PW and Jaenisch R: DNA methylation and cancer. Hum Mol Genet 3, 1487-1495, 1994.

15. Liu L, Wylie RC, Andrews LG and Tollefsbol TO: Aging, cancer and nutrition: The DNA methylation connection. Mech Ageing Dev 124: 989-998, 2003.

16. Maruyama R, Toyooka S, Toyooka KO, Harada K, Virmani AK, Zöchbauer-Müller S, Farinas AJ, Vakar-Lopez F, Minna JD, Sagalowsky A, et al: Aberrant promoter methylation profile of bladder cancer and its relationship to clinicopathological features. Cancer Res 61: 8659-8663, 2001.

17. Fabbri $M$ and Calin GA: Epigenetics and miRNAs in human cancer. Adv Genet 70: 87-99, 2010

18. Kunej T, Godnic I, Ferdin J, Horvat S, Dove P and Calin GA: Epigenetic regulation of microRNAs in cancer: An integrated review of literature. Mutat Res 717: 77-84, 2011.

19. Melo SA and Esteller M: Dysregulation of microRNAs in cancer: Playing with fire. FEBS Lett 585: 2087-2099, 2011.

20. Balaguer F, Link A, Lozano JJ, Cuatrecasas M, Nagasaka T, Boland CR and Goel A: Epigenetic silencing of miR-137 is an early event in colorectal carcinogenesis. Cancer Res 70: 6609-6618, 2010.

21. Fabbri M, Garzon R, Cimmino A, Liu Z, Zanesi N, Callegari E, Liu S, Alder H, Costinean S, Fernandez-Cymering C, et al: MicroRNA-29 family reverts aberrant methylation in lung cancer by targeting DNA methyltransferases $3 \mathrm{~A}$ and 3B. Proc Natl Acad Sci USA 104: 15805-15810, 2007.

22. Varambally S, Cao Q, Mani RS, Shankar S, Wang X, Ateeq B, Laxman B, Cao X, Jing X, Ramnarayanan K, et al: Genomic loss of microRNA-101 leads to overexpression of histone methyltransferase EZH2 in cancer. Science 322: 1695-1699, 2008.

23. Ji W, Yang L, Yuan J, Yang L, Zhang M, Qi D, Duan X, Xuan A, Zhang W, Lu J, et al: MicroRNA-152 targets DNA methyltransferase 1 in NiS-transformed cells via a feedback mechanism. Carcinogenesis 34: 446-453, 2013.

24. Qi D, Li J, Que B, Su J, Li M, Zhang C, Yang M, Zhou G and Ji W: Long non-coding RNA DBCCR1-003 regulate the expression of DBCCR1 via DNMT1 in bladder cancer. Cancer Cell Int 16: 81, 2016.

25. Huang J, Wang Y, Guo Y and Sun S: Downregulated microRNA-152 induces aberrant DNA methylation in hepatitis B virus-related hepatocellular carcinoma by targeting DNA methyltransferase 1. Hepatology 52: 60-70, 2010.

26. Livak KJ and Schmittgen TD: Analysis of relative gene expression data using real-time quantitative PCR and the $2-\Delta \Delta \mathrm{Cq}$ method. Methods 25: 402-408, 2001.

27. Wilson AS, Power BE and Molloy PL: DNA hypomethylation and human diseases. Biochim Biophys Acta 1775: 138-162, 2007.

28. Lewis BP, Shih IH, Jones-Rhoades MW, Bartel DP and Burge CB: Prediction of mammalian microRNA targets. Cell 115: 787-798, 2003.

29. Egger G, Liang G, Aparicio A and Jones PA: Epigenetics in human disease and prospects for epigenetic therapy. Nature 429: 457-463, 2004.

30. Sutherland JE and Costa M: Epigenetics and the environment. Ann NY Acad Sci 983: 151-160, 2003.

31. Tsuchiya $\mathrm{N}$ and Nakagama H: MicroRNA, SND1, and alterations in translational regulation in colon carcinogenesis. Mutat Res 693: 94-100, 2010.

32. Low SY, Ho YK, Too HP, Yap CT and Ng WH: MicroRNA as potential modulators in chemoresistant high-grade gliomas. J Clin Neurosci 21: 395-400, 2014.

33. Nugent M: MicroRNA function and dysregulation in bone tumors: The evidence to date. Cancer Manag Res 6: 15-25, 2014.

34. Gregory RI and Shiekhattar R: MicroRNA biogenesis and cancer. Cancer Res 65: 3509-3512, 2005.

35. Stumpel DJ, Schotte D, Lange-Turenhout EA, Schneider P, Seslija L, de Menezes RX, Marquez VE, Pieters R, den Boer ML and Stam RW: Hypermethylation of specific microRNA genes in MLL-rearranged infant acute lymphoblastic leukemia: Major matters at a micro scale. Leukemia 25: 429-439, 2011. 
36. Miao CG, Qin D, Du CL, Ye H, Shi WJ, Xiong YY, Zhang XL Yu H, Dou JF, Ma ST, et al: DNMT1 activates the canonical Wnt signaling in rheumatoid arthritis model rats via a crucial functional crosstalk between miR-152 and the DNMT1, MeCP2. Int Immunopharmacol 28: 344-353, 2015.

37. Zhu C, Li J, Ding Q, Cheng G, Zhou H, Tao L, Cai H, Li P, Cao Q, $\mathrm{Ju} \mathrm{X}$, et al: $\mathrm{miR}-152$ controls migration and invasive potential by targeting TGF $\alpha$ in prostate cancer cell lines. Prostate 73 1082-1089, 2013.

38. Cheng Z, Ma R, Tan W and Zhang L: MiR-152 suppresses the proliferation and invasion of NSCLC cells by inhibiting FGF2. Exp Mol Med 46: e112, 2014.

39. Ma J, Yao Y, Wang P, Liu Y, Zhao L, Li Z, Li Z and Xue Y: MiR-152 functions as a tumor suppressor in glioblastoma stem cells by targeting Kruppel-like factor 4. Cancer Lett 355: 85-95, 2014.

40. Chen S, Sanjana NE, Zheng K, Shalem O, Lee K, Shi X, Scott DA, Song J, Pan JQ, Weissleder R, et al: Genome-wide CRISPR screen in a mouse model of tumor growth and metastasis. Cell 160: 1246-1260, 2015

41. Xu Q, Jiang Y, Yin Y, Li Q, He J, Jing Y, Qi YT, Xu Q, Li W, Lu B, et al: A regulatory circuit of miR-148a/152 and DNMT1 in modulating cell transformation and tumor angiogenesis through IGF-IR and IRS1. J Mol Cell Biol 5: 3-13, 2013.

42. Zheng B, Liang L, Wang C, Huang S, Cao X, Zha R, Liu L, Jia D, Tian Q, Wu J, et al: MicroRNA-148a suppresses tumor cell invasion and metastasis by downregulating ROCK1 in gastric cancer. Clin Cancer Res 17: 7574-7583, 2011.

43. Nakagawa T, Kanai Y, Ushijima S, Kitamura T, Kakizoe T and Hirohashi S: DNA hypermethylation on multiple $\mathrm{CpG}$ islands associated with increased DNA methyltransferase DNMT1 protein expression during multistage urothelial carcinogenesis. J Urol 173: 1767-1771, 2005.
44. Szyf M and Detich N: Regulation of the DNA methylation machinery and its role in cellular transformation. Prog Nucleic Acid Res Mol Biol 69: 47-79, 2001.

45. Xiang Y, Ma N, Wang D, Zhang Y, Zhou J, Wu G, Zhao R, Huang $\mathrm{H}$, Wang $\mathrm{X}$, Qiao $\mathrm{Y}$, et al: MiR-152 and miR-185 co-contribute to ovarian cancer cells cisplatin sensitivity by targeting DNMT1 directly: A novel epigenetic therapy independent of decitabine. Oncogene 33: 378-386, 2014.

46. Miao CG, Yang YY, He X, Huang C, Huang Y, Qin D, Du CL and Li J: MicroRNA-152 modulates the canonical Wnt pathway activation by targeting DNA methyltransferase 1 in arthritic rat model. Biochimie 106: 149-156, 2014.

47. Wiklund ED, Kjems J and Clark SJ: Epigenetic architecture and miRNA: Reciprocal regulators. Epigenomics 2: 823-840, 2010.

48. Sato F, Tsuchiya S, Meltzer SJ and Shimizu K: MicroRNAs and epigenetics. FEBS J 278: 1598-1609, 2011.

49. Azizi M, Teimoori-Toolabi L, Arzanani MK, Azadmanesh K, Fard-Esfahani $P$ and Zeinali S: MicroRNA-148b and microRNA-152 reactivate tumor suppressor genes through suppression of DNA methyltransferase-1 gene in pancreatic cancer cell lines. Cancer Biol Ther 15: 419-427, 2014.

50. Wang J, Bian Y, Wang Z, Li D, Wang C, Li Q and Gao X: MicroRNA-152 regulates DNA methyltransferase 1 and is involved in the development and lactation of mammary glands in dairy cows. PLoS One 9: e101358, 2014.

51. Cui H, Wang L, Gong P, Zhao C, Zhang S, Zhang K, Zhou R, Zhao Z and Fan H: Deregulation between miR-29b/c and DNMT3A is associated with epigenetic silencing of the CDH1 gene, affecting cell migration and invasion in gastric cancer. PLoS One 10: e0123926, 2015. 